\title{
La incorporación de los departamentos de comuni- cación de las universidades españolas al entorno digital. Un análisis cuantitativo
}

\author{
Francisco Javier Paniagua RoJANO \\ Universidad de Málaga \\ fjpaniagua@uma.es \\ Bernardo J. Gómez CALDERóN \\ Universidad de Málaga \\ bjgomez@uma.es \\ Manuel FERNÁNDEZ SANDE \\ Universidad Complutense de Madrid \\ manuel.fernandez@ccinf.ucm.es
}

Recibido: $31 / 07 / 2012$

Aceptado: 24/10/2012

\section{Resumen}

El objetivo del presente trabajo consiste en determinar el grado de adaptación de los departamentos de comunicación de las universidades españolas al entorno web 2.0, y comprobar si, a través de él, atienden por igual a sus públicos internos y externos. Para ello, se han analizado sus salas de prensa virtuales y las herramientas on line que utilizan de modo más frecuente, así como su incorporación a las redes sociales y el nivel de interactuación que despliegan en ellas.

Los resultados revelan una progresiva implantación de recursos interactivos entre los gabinetes universitarios, aunque aún son mayoría los que se limitan a trasladar sin modificaciones los contenidos que producen de forma tradicional, al tiempo que se evidencia cierto desequilibrio en la atención prestada a los distintos tipos de públicos.

Palabras clave: Comunicación institucional, gabinetes de prensa, web 2.0, redes sociales.

\section{The Incorporation of the Spanish Universities' Press offices to the Digital Environment. A Quantitative Analysis}

\begin{abstract}
The aim of the present study has been to analyze the incorporation of the Spanish universities' press offices to the Web 2.0, checking if they attend equally to the internal and external users. In order to achieve this, we have focused on specific features presented by their offices and their most frequently used on line communication resources, as well as their incorporation to the social networks and their degree of interaction.

The analysis has shown that press offices have undertaken a progressive adaptation to the digital environment; although, the method used by the majority of these offices to disseminate the material remains traditional, i.e. without further modifications. Besides, we have detected than some users get more attention than others.
\end{abstract}

Key words: Institutional communication, press offices, Web 2.0, social networks.

\section{Referencia normalizada}

PANIAGUA ROJANO, Francisco Javier; GÓMEZ CALDERÓN, Bernardo J. y FERNÁNDEZ SANDE, Manuel (2012): "La incorporación de los departamentos de comunicación de las universidades españolas al entorno digital. Un análisis cuantitativo". Estudios sobre el mensaje periodístico. Vol. 18, núm. especial noviembre, págs.: 691-701. Madrid, Servicio de Publicaciones de la Universidad Complutense.

Sumario: 1. Introducción; 1.1. La comunicación en la Universidad, hoy; 1.2. La web 2.0 y los departamentos de comunicación. 2. Metodología. 3. Desarrollo; 3.1. Tipos de gabinetes; 3.2. Principales herra- 
mientas utilizadas; 3.2.1. La nota de prensa 2.0 o interactiva; 3.3. Integración de las redes sociales en la estrategia de comunicación de las universidades; 3.3.1. Las universidades españolas en Facebook; 3.3.2. Las universidades españolas en Twitter; 3.3.3. Las universidades españolas en Linkedin; 3.3.4. El uso de Youtube por parte de las universidades; 3.4. Valoración del nivel de interactuación. 4. Conclusiones; 5. Referencias bibliográficas

\section{Introducción}

Partiendo del convencimiento de que toda estrategia de comunicación institucional se basa en lo que el profesor Farias Batlle denomina el "Triángulo de la Comunicación" (2000: 65), entendemos que cualquier organización tiene la necesidad de mantenerse en contacto con sus públicos internos, emitiendo y recibiendo mensajes de manera planificada, para conseguir que estos tengan una imagen positiva de su misión y funcionamiento (la denominada "cultura institucional"). En el caso de las universidades, dichos públicos los conforman sus empleados, estudiantes, profesores e investigadores, antiguos alumnos, personal de administración y servicios y representantes de los trabajadores.

Al mismo tiempo y con idéntico propósito, las organizaciones precisan del intercambio de mensajes con sus públicos externos, que en el ámbito universitario engloban a estudiantes potenciales, empresas del entorno, sociedad, gobiernos, otros organismos de enseñanza superior, centros escolares, consejos sociales, proveedores y medios de comunicación. De este modo se labra la "imagen corporativa".

Sólo cuando se consigue un equilibrio entre ambas esferas de actividad, la Universidad logra proyectar una imagen institucional óptima, sobre todo si es capaz de controlar los condicionantes externos -o interacciones- que pueden alterar los flujos de información (véase gráfico 1).

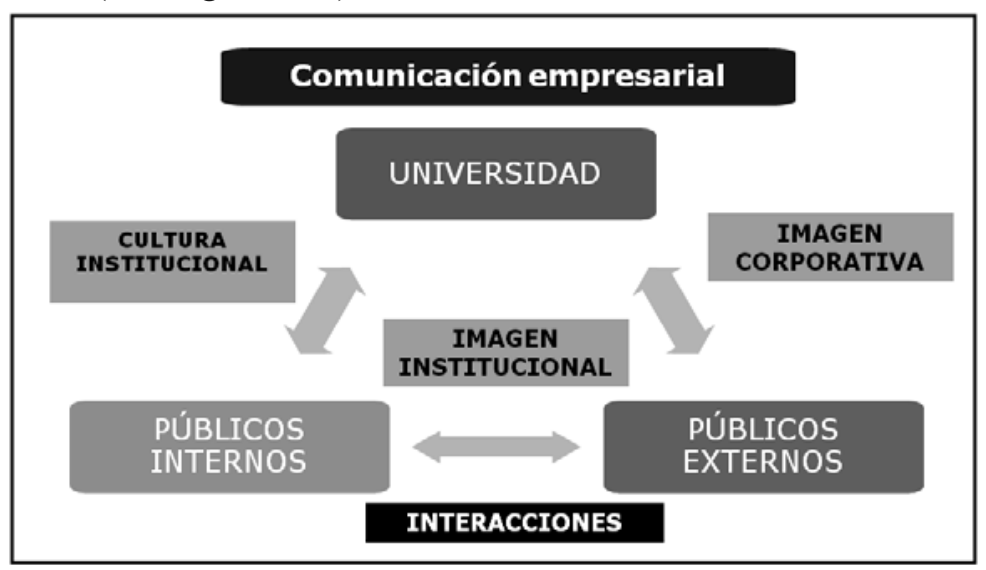

Gráfico 1. El "Triángulo de la Comunicación" aplicado a la Universidad. Fuente: Farias Batlle, 2000. Elaboración propia.

En este circuito se ha introducido, durante el último decenio, un aliado de considerable potencial, por su capacidad de retroalimentación y la versatilidad de sus contenidos: Internet. Y la aplicación de las herramientas asociadas a la web ha traído consigo una modificación de las rutinas comunicativas cuyo impacto se está dejando ya sentir. 


\subsection{La comunicación en la Universidad, hoy}

La Universidad atraviesa actualmente un periodo de transformaciones profundas, propiciado por la convergencia educativa a escala europea (el conocido como "proceso de Bolonia") y el incremento de la competitividad en un mercado con tendencia a la saturación (cf. Chapleo, 2004). Cada institución tiene la necesidad de presentar ante sus públicos una oferta claramente diferenciada, condición sin la cual "su supervivencia continuada se verá seriamente comprometida" (Losada, 2004: 476).

Las labores de comunicación han adquirido, por ello, una trascendencia singular en el diseño de las políticas universitarias.

No fue hasta la llegada de la democracia cuando comenzaron a impulsarse los primeros servicios de prensa en los campus españoles. Su objetivo era lograr cierta proyección pública a través de los medios de comunicación, algo a lo que hasta la fecha no se había prestado atención. En el plazo de dos décadas, los primitivos gabinetes lograron consolidarse como instrumentos fundamentales de la organización universitaria, y junto a las relaciones con la prensa, los responsables del servicio fueron asumiendo cometidos que implicaban también a los públicos internos -desde los estudiantes hasta el personal de administración- e impulsando actividades vinculadas a las nuevas tecnologías.

Pero a pesar del desarrollo conseguido, la estructura y el cometido de los gabinetes universitarios españoles transmiten aún una imagen poco uniforme. En muchos casos, se plantean como meros instrumentos al servicio de los agentes externos, principalmente los medios de comunicación, desatendiendo a los colectivos de la propia universidad (Palencia-Lefler Ors, 2008), a pesar de la importancia que el flujo de información hacia estudiantes y profesores ha adquirido en el actual contexto de reforma, como apuntan Herranz, Tapia y Vicente (2008).

Probablemente tenga razón Fernández Beltrán (2007: 552) cuando afirma que en este terreno se produce una frecuente dispersión de funciones, achacable en buena medida a la falta de compromiso de los órganos de dirección. Y aunque se detecta una tendencia creciente al establecimiento de áreas específicas de comunicación-que principalmente se sitúan en la órbita de las Relaciones Institucionales-, son pocos los casos en los que estas competencias recaen en vicerrectorados creados ex profeso'.

\subsection{La web 2.0 y los departamentos de comunicación}

La irrupción de la comunicación 2.0, apoyada en aplicaciones y canales que permiten una elevada interactividad entre los usuarios (Cebrián, 2008), se está dejando sentir en la gestión informativa de las universidades, pues ofrece la posibilidad de establecer una relación directa con los públicos internos y externos sin el filtro de los medios convencionales (cf. San Millán, Blanco y Del Arco, 2008).

Entre las herramientas 2.0 sobresalen las redes sociales, de indudable interés para cualquier institución educativa. De acuerdo con Regalado (2011), su utilización estratégica puede servir, entre otras cosas, para construir una imagen de marca asociada a valores positivos y para incrementar la comunicación con la comunidad estudiantil.

1 En la actualidad, sólo se localizan en las universidades de Barcelona, Córdoba, Jaén, Málaga, Navarra y Zaragoza. 
En el ámbito universitario su integración ha sido veloz (cf. Celaya y Herrero, 2007), siguiendo la estela de los campus norteamericanos, que hacen de este tipo de aplicaciones un uso intensivo y planificado, tal y como ponen de manifiesto Herreros (2010) y Ganim y Lescault (2011).

Las nuevas herramientas informáticas están propiciando la transformación de los gabinetes de prensa tradicionales en servicios de carácter digital. De acuerdo con García Orosa (2009: 23), el departamento on line es aquel que realiza total o parcialmente su actividad en la Red. Los principios que rigen su actuación no varían con respecto a la comunicación institucional clásica, pero su aplicación se produce en un nuevo escenario marcado por la inmediatez, la interactividad y la retroalimentación.

En función de su grado de adecuación al entorno telemático, la propia García Orosa (2009: 25-27) propone una clasificación que consideramos de utilidad: gabinetes on line 1.0 (sólo incluyen datos sobre el departamento y un correo electrónico de contacto), gabinetes on line 1.1 (ofrecen noticias sobre la organización sin actualización periódica, compuestas únicamente por texto y/o imágenes estáticas), gabinetes on line 1.2 (vuelcan el material que produce el área de prensa, en especial notas, convocatorias y dosieres, de forma sistemática pero con escasos elementos añadidos) y gabinetes 2.0 (comunidades colaborativas en las que se crean estructuras de participación y se promueve el feedback de los periodistas y otros públicos de interés).

El modelo más habitual, a día de hoy, es el 1.2, pero cada vez son más los departamentos universitarios que incorporan a su oferta de servicios las potencialidades de la web 2.0, como se verá a continuación.

\section{Metodología}

El objetivo fundamental del presente trabajo consistía en analizar la incorporación de herramientas digitales a las labores de comunicación de las universidades españolas, con el propósito último de determinar si, a través de ellas, atienden por igual a sus públicos internos y externos y exhiben, de este modo, una imagen equilibrada.

La muestra del estudio la componen las 78 universidades que en la actualidad funcionan en nuestro país. De cada una se analizó, entre el 20 y el 26 de julio de 2012, su página web, en concreto la sala de prensa o sección de comunicación, identificando el tipo de gabinete digital que mantienen y su adecuación al entorno 2.0, así como las herramientas de comunicación on line a las que recurren de modo más frecuente. De igual modo, establecimos su integración con las redes sociales, las estrategias aplicadas en ellas y el predominio o equilibrio de actuaciones internas y externas, en una ficha que incluía también una escala de valoración a propósito del nivel de interactuación logrado por cada institución.

\section{Desarrollo}

La oferta de comunicación digital de las universidades españolas resulta notablemente heterogénea, tanto por la estructura de sus gabinetes como por los recursos que ponen a disposición de los usuarios y por su actividad en las redes sociales. Desde el simple suministro de información de contacto y noticias de interés hasta el desarrollo de verdaderas salas de prensa virtuales, el abanico de soluciones desplegado es amplio, y su grado de efectividad, diverso. 


\subsection{Tipos de gabinetes}

Coincidimos con García Orosa (2009: 27) en que la mayoría de los departamentos institucionales adoptaron Internet como un canal de información y no de comunicación. Es lo que se deduce en el caso de las universidades españolas. Atendiendo a la clasificación de esta autora, más de la mitad de los servicios (57\%) se pueden considerar gabinetes 1.2, ya que se limitan a volcar documentos creados para su difusión por otros canales. Un $20 \%$ todavía cuenta en su página web con un departamento 1.1, dedicado sólo a difundir noticias de interés; pero casi la cuarta parte, un $23 \%$, ha organizado ya una comunidad virtual en la que el intercambio de información se produce de manera bidireccional.

Así, encontramos que 17 de las 78 universidades analizadas disponen de un espacio 2.0: se trata de las de Abat Oliba-CEU, Alicante, Autónoma de Barcelona, Barcelona, Camilo José Cela, Cardenal Herrera-CEU, Católica Santa Teresa de Jesús, Córdoba, Francisco de Vitoria, Granada, Huelva, La Laguna, Mondragón, Navarra, Politécnica de Catalunya, Sevilla y UNED.

\subsection{Principales herramientas utilizadas}

La mayoría de las universidades incluyen en su sala de prensa notas y comunicados $(96,15 \%)$, dosieres $(66,67 \%)$, vídeos $(62,82 \%)$ y fotografías $(57,69 \%)$. Únicamente un $48,72 \%$ pone a disposición de los usuarios servicios de seguimiento de medios, habitualmente prestados por agencias externas. Otras herramientas trasladadas con frecuencia del gabinete tradicional al digital son la revista interna $(34,62 \%)$ y el informe anual $(11,54 \%)$. Como puede apreciarse, se trata de productos escasamente innovadores desde el punto de vista telemático.

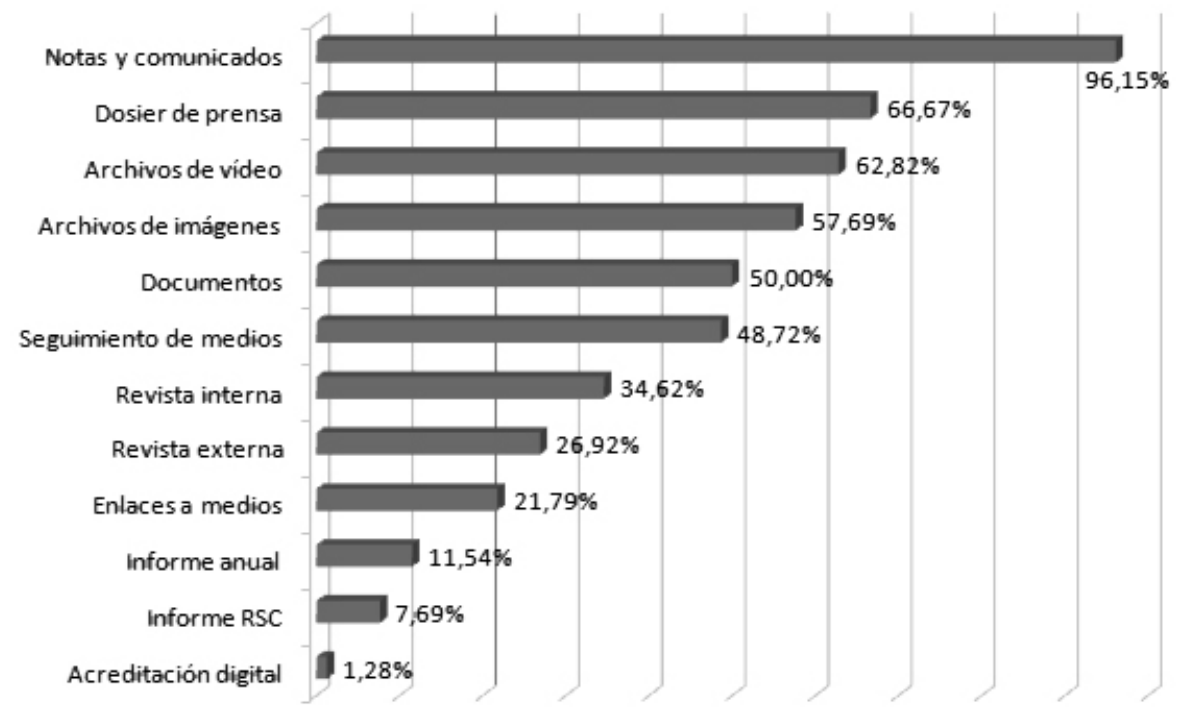

Gráfico 2. Herramientas de comunicación más comunes en las webs de las universidades.

Fuente: webs de universidades españolas. Elaboración propia. 


\subsubsection{La nota de prensa 2.0 o interactiva}

Uno de los instrumentos más atractivos que permite el entorno digital es la nota $2.0 \mathrm{o}$ interactiva. De acuerdo con Carreras (2010), se trata de un documento que, junto al texto tradicional, incorpora recursos y aplicaciones surgidas en Internet, como podcasts, RSS o hipertextos. Hay que tener en cuenta que la audiencia, a la que antes se llegaba a través de los medios de comunicación masivos, se ha disgregado en colectividades on line profundamente segmentadas, y sus necesidades informativas, como advierte Carreras, han evolucionado.

Pero pese a su idoneidad, llama la atención que los gabinetes de comunicación de las universidades españolas no hayan apostado todavía de manera decidida por las notas interactivas en sus páginas web. Aunque un $44,87 \%$ ofrece la posibilidad de compartir archivos en las redes sociales, sólo el 20,51\% elabora documentos 2.0 , acompañados de enlaces a contenidos adicionales o páginas de interés.

\subsection{Integración de las redes sociales en la estrategia de comunicación de las uni- versidades}

Hace dos años, más del 70\% de las universidades españolas estaban presentes en alguna de las redes sociales (Aguilera, Farias y Baraybar, 2010). Hoy, esa proporción ha crecido hasta el 96,15\%. La mayoría dispone de página en Facebook (68, lo cual equivale al $87 \%$ del total), y casi las mismas tienen perfil de Twitter (67, esto es, un $86,9 \%$ ). Además, 66 poseen un espacio en Youtube, 46 mantienen página de empresa en Linkedin, 31 aparecen en Tuenti y 28 usan Flickr para alojar fotografías en red. Las instituciones analizadas sumaban a 26 de julio de 2012 un total de 849.428 seguidores entre Facebook, Twitter, Linkedin y Youtube, las aplicaciones más populares.

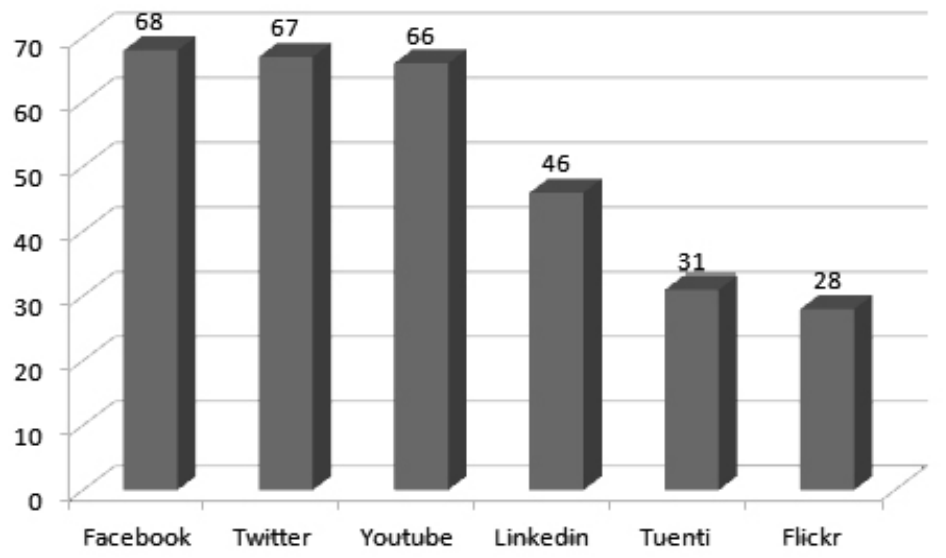

Gráfico 3. Presencia de las universidades españolas en las redes sociales. Fuentes: webs de universidades, redes sociales. Elaboración propia.

Un 75\% de la muestra identifica claramente su presencia en las redes, siendo el lugar elegido, en la mayoría de los casos, la portada de la web (56 ocasiones), seguida 
de la sala de prensa (11). Sólo ocho departamentos cuentan con una sección especial dedicada a las social networks, los de las universidades de Alcalá, Alicante, Carlos III, Complutense, Málaga, Menéndez Pelayo, Rey Juan Carlos y Sevilla.

\subsubsection{Las universidades españolas en Facebook}

Facebook aloja al 87,18\% de las 78 universidades españolas. El perfil de su público, compuesto por 375.607 fans, es predominantemente interno, con mayoría de estudiantes. Los eventos y noticias que se comparten en esta red, sin embargo, no generan excesiva retroalimentación, como prueba el hecho de que, durante el mes analizado, únicamente se registraran 7.981 comentarios, una cifra que se antoja baja si tenemos en cuenta la rápida interactividad que caracteriza al canal.

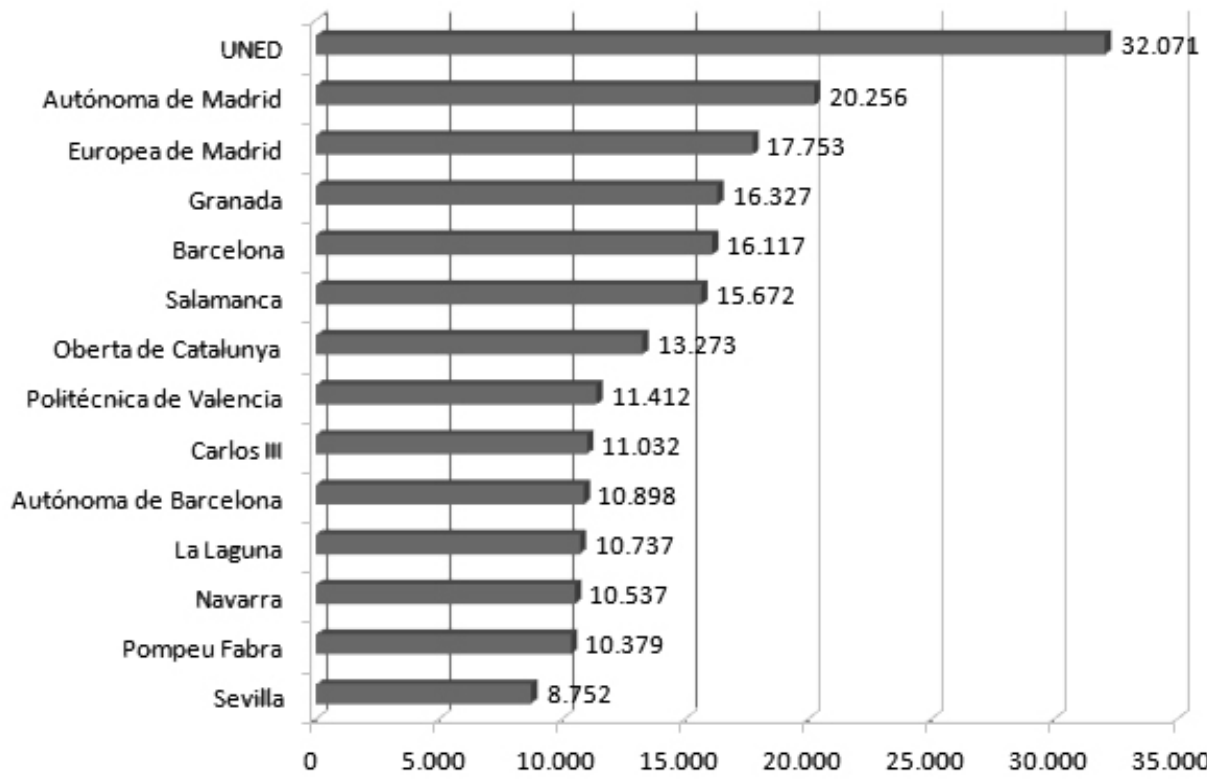

Gráfico 4. Universidades con mayor número de fans en Facebook. Fuente: Facebook. Elaboración propia.

Quince instituciones acaparan más del 56\% del total de seguidores en Facebook (véase gráfico 4), aunque sólo algunas de ellas generan una respuesta significativa entre los usuarios. En este sentido, sobresalen la UNED, con 1.339 comentarios en julio de 2012; València-Estudi General, con 606; y Granada, con 511.

\subsubsection{Las universidades españolas en Twitter}

Como se ha apuntado, un $86,9 \%$ de las universidades implantadas en nuestro país cuentan con perfil institucional en Twitter, sumando un total de 379.692 seguidores, identificados, sobre todo, como estudiantes, profesores y profesionales de los medios de comunicación. A 26 de julio de 2012, el volumen global de tuits ascendía a 162.745. 


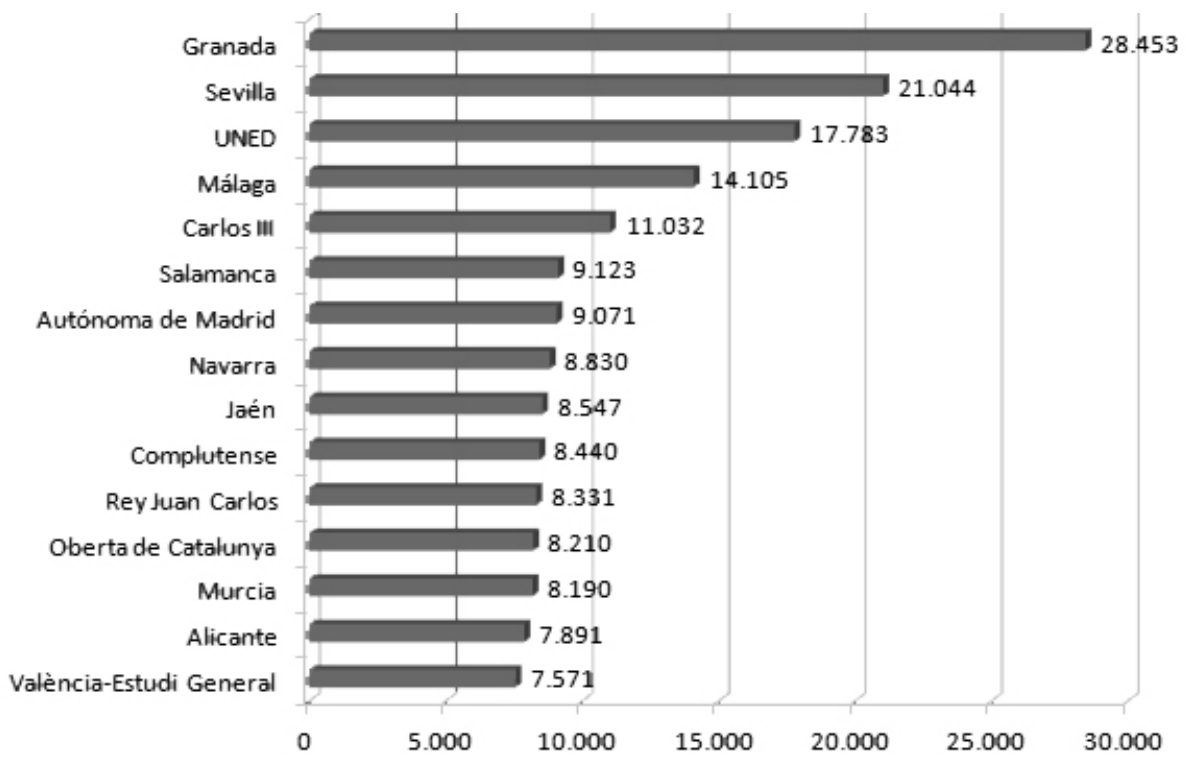

Gráfico 5. Universidades con más seguidores en Twitter. Fuente: Twitter. Elaboración propia.

No obstante, en conjunto sólo siguen a 66.085 perfiles en esta red, lo cual revela una notable debilidad comunicativa, ya que la atención a la actividad de otras personas e instituciones constituye una práctica valiosa a través de la cual tomar el pulso a los públicos internos y externos y, de este modo, detectar corrientes de opinión que ayuden a planificar nuevas estrategias comunicativas.

Además, del análisis de los mensajes generados se deduce que el principal uso que se da a Twitter es la simple difusión de notas de prensa y eventos, en detrimento del establecimiento de procesos dialógicos que serían más provechosos para la institución y más satisfactorios para los usuarios.

\subsubsection{Las universidades españolas en Linkedin}

A pesar de ser una aplicación de carácter profesional que ofrece la posibilidad de desarrollar proyectos colaborativos, encontrar empleo o captar personal cualificado, Linkedin figura como la cuarta red social para las universidades españolas; sólo 46 de ellas la emplean, con un volumen de seguidores de 71.182. No obstante, a estos hay que añadir los grupos, de los que funcionan más de 500, especialmente de antiguos alumnos, centros, departamentos y proyectos de investigación. Si estos grupos son conocidos por la dirección de comunicación y se siguen estrategias para mejorar la interacción con ellos, resulta difícil de determinar.

De nuevo, 15 de las 46 universidades con presencia en Linkedin acaparan más del $70 \%$ de los usuarios. Destacan, en este sentido, la Complutense de Madrid (6.579), la de Barcelona (5.576), la Politécnica de Madrid (5.010), la Europea de Madrid (3.579) y la de Sevilla (3.530). En el mismo orden, las que disponen de más grupos de discusión son la Complutense, la Politécnica de Madrid, la de Barcelona, la de Sevilla, la de Salamanca, la Carlos III y la Rey Juan Carlos. 


\subsubsection{El uso de Youtube por parte de las universidades}

Youtube está adquiriendo cada vez mayor peso como portal a través del cual proporcionar una dimensión audiovisual a la actividad de las instituciones universitarias. De ahí que el número de vídeos compartidos por éstas hasta el 26 de julio de 2012 ascienda a 18.163, y el total de suscriptores se cifre en 22.947.

Las universidades más activas en Youtube son la Politécnica de Valencia (2.192 piezas), la Oberta de Catalunya (1.978), la Politécnica de Madrid (1.593), la UNED (812), la de Navarra (725) y la de Vigo (580). Entre el material difundido predominan los vídeos corporativos, aunque van ganando terreno las producciones orientadas a la divulgación del conocimiento, siempre en forma de conferencias o clases magistrales.

\subsection{Valoración del nivel de interactuación}

Para finalizar, y una vez analizada la oferta comunicativa on line de las universidades españolas, procedimos a valorar su grado de interactuación con los públicos de interés tanto internos como externos, a partir del tipo de contenidos que suministran y la retroalimentación que propician. En una escala del 1 al 5, siendo 1 "escasamente interactiva" y 5 "altamente interactiva", la mayoría de las instituciones alcanzan el aprobado, aunque con una media discreta $(2,59)$.

Para los autores, las universidades cuidan en mayor medida la comunicación interna, en especial la destinada a estudiantes y profesores, que la dirigida a medios, empresas o sociedad en general. En el primer caso, el promedio valorativo se sitúa en 2,54 , mientras que en el segundo, la escasez de herramientas interactivas puestas a disposición de los periodistas reduce el índice a 2,44.

\section{Conclusiones}

Nuestro análisis constata la progresiva adaptación de los departamentos de comunicación de las universidades españolas al entorno 2.0, aunque aún son mayoría (77\%) los que se limitan a trasladar sin modificaciones los materiales que producen de forma tradicional, generando escaso feedback entre sus públicos internos y externos.

Los gabinetes on line funcionan más como salas de prensa que como verdaderos espacios de comunicación. Todos ofrecen a través de Internet noticias de actualidad y una vía de contacto; en algunas ocasiones, también la agenda de la semana, comunicados y dosieres sobre temas de interés; pero en realidad, no se trata de nada que no pueda facilitar un departamento tradicional. Rara vez se aprovechan las ventajas de la comunicación digital como el hipertexto, los archivos multimedia, la interactividad o la clasificación de las informaciones por criterios temáticos o territoriales.

El tipo de materiales que los gabinetes incorporan resulta, en muchos casos, una simple réplica de su producción en papel. Herramientas novedosas como la nota de prensa 2.0 se detecta sólo en un $20,51 \%$ de los casos.

Por otro lado, la práctica totalidad de las universidades españolas -un 96,15\%cuenta con perfiles en Facebook, Twitter, Youtube o Linkedin. Sin embargo, esta presencia todavía no genera un rendimiento comunicativo óptimo, ya que con frecuencia las redes se emplean con el único fin de difundir notas de prensa y compartir eventos, sin incentivar la participación de los seguidores. Esto, unido al escaso número 
de comentarios generados, nos lleva a pensar que la actividad desplegada en las social networks carece de una planificación estratégica eficaz.

Finalmente, la valoración general de las tareas de comunicación llevadas a cabo por las universidades a través de sus páginas web refleja cierta descompensación entre la atención prestada a los usuarios internos y externos, siendo mejor atendidos los primeros. Esto se traduce en un Triángulo de la Comunicación desequilibrado, que no desarrolla convenientemente algunas de las funciones que tiene asignadas, lo cual entendemos que resulta perjudicial para la imagen pública de las instituciones analizadas.

\section{Referencias bibliográficas}

AGUILERA MOYANO, Miguel; FARIAS BATLLE, Pedro y BARAYBAR, Antonio (2010): "La comunicación universitaria: modelos, tendencias y herramientas para una nueva relación con sus públicos". Icono 14, año 8, vol. 2. Madrid, Asociación Científica de Comunicación y Nuevas Tecnologías, pp. 90-124. Disponible en: http://www.icono14.net/Num.-16.-Nuevos-tiempos-para-la-comunicacion/lacomunicacion-universitaria [fecha de consulta: 12 de julio de 2012].

CARRERAS, Roberto (2010): "Las notas de presa ante el nuevo escenario 2.0", en: $\mathrm{http} / / /$ robertocarreras.es/las-notas-de-prensa-ante-el-nuevo-escenario-2-0/ [fecha de consulta: 16 de mayo de 2012].

CEBRIÁN HERREROS, Mariano (2008): "La Web 2.0 como red social de información y comunicación". Estudios sobre el Mensaje Periodístico, vol. 14, Madrid, Servicio de Publicaciones de la Editorial Complutense, pp. 345-361. Disponible en http://revistas.ucm.es/index.php/ESMP/article/view/ESMP0808110345A/11928 [fecha de consulta: 24 de septiembre de 2012].

CELAYA, Javier y HERRERA, Pau (2007): Comunicación empresarial 2.0. La función de las nuevas tecnologías sociales en la estrategia de comunicación empresarial. Barcelona, Grupo BPMO.

CHAPLEO, Christopher (2004): "Interpretation and implementation of reputation/brand management by UK University leaders". International Journal of Educational Advancement, $\mathrm{n}^{\circ} 5$ (1). Londres, Palgrave Mcmillan, pp. 7-23.

FARIAS BATLLE, Pedro (2000): Empresa informativa. Proyecto docente inédito. Málaga, Universidad de Málaga.

FERNÁNDEZ BELTRÁN, Francisco (2007): La gestión de la nueva comunicación interna. Análisis de la aplicación de las tecnologías de la información en los procesos de comunicación interna de las universidades de la Comunidad Valenciana. Tesis doctoral inédita. Alicante, Universitat Jaume I.

GANIM, Nora y LESCAULT, Ava (2011): "Social media adoption soars as highersed experiments and reevaluates its use of new communication tools" en: http://www.umassd.edu/media/umassdartmouth/cmr/studiesandresearch/higherEd. pdf [fecha de consulta: 3 de junio de 2012]. 
GARCÍA OROSA, Berta (2009): Gabinetes de comunicación on line. Sevilla-Salamanca-Zamora, Comunicación Social Ediciones y Publicaciones.

HERRANZ DE LA CASA, José María; TAPIA FRADE, Alejandro; y VICENTE LÁZARO, Arturo (2009): "La comunicación interna en la universidad. Investigar para conocer a nuestros públicos". Revista Latina de Comunicación Social, n 64, pp. 262-274. Disponible en http://www.ull.es/publicaciones/latina/09/art/23_822_30 _Valladolid/Herranz_et_al.html [fecha de consulta: 13 de junio de 2012].

HERREROS, Pablo (2010): "Social Media en universidades", en: http://comunicacionsellamaeljuego.com/ejemplos-de-social-media-en-universidades/ [fecha de consulta: 25 de junio de 2012].

LOSADA DÍAZ, José Carlos (Coord. 2004): Gestión de la comunicación en las organizaciones. Barcelona, Ariel.

PALENCIA-LEFLER ORS, Manuel (2008): "La incomunicación interna en la Universidad española". Revista Latina de Comunicación Social, no 63, pp. 277-286. Disponible en http://www.ull.es/publicaciones/latina/_2008/22_36_UPF/Manel _Palencia.html [fecha de consulta: 29 de junio de 2012].

REGALADO, Octavio (2011): “¿Cómo deberían usar las universidades las redes sociales?", en: http://www.dosensocial.com/category/dosensocial [fecha de consulta: 30 de mayo de 2012].

SAN MILLÁN, Elvira; BLANCO, Francisco y DEL ARCO, José Carlos (2008): “Comunicación corporativa 2.0 en la Universidad Rey Juan Carlos", en DE CASTRO, Emilio y DÍAZ, José (coords.): Universidad, sociedad y mercados globales. Madrid, ESIC, pp. 394-408.

\section{Francisco Javier PANIAGUA ROJANO}

Profesor Contratado Doctor del Departamento de Periodismo de la Universidad de Málaga fjpaniagua@uma.es

\section{Bernardo J. GÓMEZ CALDERÓN}

Profesor Contratado Doctor del Departamento de Periodismo de la Universidad de Málaga bjgomez@uma.es

Departamento de Periodismo

Facultad de Ciencias de la Comunicación. Universidad de Málaga

Campus de Teatinos, s/n - 29071 Málaga

\section{Manuel FERNÁNDEZ SANDE}

Profesor Contratado Doctor del Departamento de Periodismo IV de la UCM manuel.fernandez@ccinf.ucm.es Departamento de Periodismo IV Facultad de Ciencias de la Información. Universidad Complutense de Madrid Ciudad Universitaria - 28040 Madrid 